\title{
On Optimal Single-Item Auctions
}

\author{
[Extended Abstract] ${ }^{*}$
}

\author{
Christos Papadimitriou \\ Computer Science Division \\ University of California at Berkeley \\ CA, 94720 USA \\ christos@cs.berkeley.edu
}

\author{
George Pierrakos \\ Computer Science Division \\ University of California at Berkeley \\ CA, 94720 USA \\ georgios@cs.berkeley.edu
}

\begin{abstract}
We revisit the problem of designing the profit-maximizing single-item auction, solved by Myerson in his seminal paper for the case in which bidder valuations are independently distributed. We focus on general joint distributions, seeking the optimal deterministic incentive compatible auction. We give a geometric characterization of the optimal auction through a duality theorem, resulting in an efficient algorithm for finding the optimal deterministic auction in the two-bidder case and an inapproximability result for three or more bidders.
\end{abstract}

\section{Categories and Subject Descriptors}

J.4 [Social and Behavioral Sciences]: Economics; F.2 [Analysis of Algorithms and Problem Complexity]: General

\section{General Terms}

Algorithms, Economics, Theory

\section{Keywords}

Bayesian optimal mechanism design, correlated valuations

\section{INTRODUCTION}

Myerson's paper [20] on optimal auction design is remarkable in several ways. It is not the first important paper on auctions of course [29], but it pioneers the point of view of the title: auction design, that is, the exploration and evaluation of a large design space in a mindset that is very much one of computer science. It is elegant and methodologically original and powerful, completely resolving this important and difficult problem and providing insights, lemmata, and methodology that would be useful in related contexts for

${ }^{*}$ A full version of this paper is available at
http://arxiv.org/abs/1011.1279
http://arxiv.org/abs/1011.1279

Permission to make digital or hard copies of all or part of this work for personal or classroom use is granted without fee provided that copies are not made or distributed for profit or commercial advantage and that copies bear this notice and the full citation on the first page. To copy otherwise, to republish, to post on servers or to redistribute to lists, requires prior specific permission and/or a fee.

STOC'11, June 6-8, 2011, San Jose, California, USA.

Copyright 2011 ACM 978-1-4503-0691-1/11/06 ...\$10.00. decades. Myerson considers the auction of an item to bidders with private valuations whose prior distributions are independent and known, and seeks incentive compatible mechanisms that maximize auctioneer revenue. His simple and elegant solution involves the virtual valuation, a quantity each bidder can compute from their private valuation and the valuation distribution through a mathematical maneuver called ironing. Myerson leaves open the case in which the valuations are correlated; in subsequent work, Crémer and McLean $[8,7]$ consider this general correlated case and solve it for the case where auctions are only required to be interim individually rational, i.e. when an agent with knowledge of their own value expects non-negative utility assuming other agents' values are drawn from a known distribution. Surprisingly, they show that the uncorrelated case is a singularity in the sense that in most cases (when the correlation has "full rank" in a certain sense) full surplus can be extracted in expectation through appropriate offers of lotteries to the bidders. Despite the elegance of their result, the fact that bidders may be charged merely for participating in the auction-lottery (including losers) has been criticized by economists as rendering the auction impractical [19], especially for settings where agents may easily cancel their participation after the auction is conducted. It is therefore of tantamount importance to consider the question of designing the optimal ex post individually rational auction for correlated valuations. This is the question we consider, and in a sense completely answer, in the current paper.

This past decade saw the advent and rise of Algorithmic Game Theory [23, 21], a research tradition which can be seen as a complexity-theoretic critique of Mathematical Economics, with Internet in the backdrop. This point of view has yielded a host of important results and new insights, for example related to the complexity of equilibria $[6$, 9], the trade-offs between complexity, approximation, and incentive-compatibility in social welfare-maximizing mechanism design [21, 24], and (in an extended sense that includes on-line algorithms as a part of complexity theory) the price of anarchy [18, 28, 27]. However, there has been little progress in looking at Bayesian auctions à-la Myerson from this point of view. Ronen [25] came up with a mechanism for the correlated case that achieves half of the optimum revenue, while Ronen and Saberi [26] showed that no "ascending auction" can do better than $7 / 8$, and they conjectured that all relevant auctions are ascending (incidentally, we disprove this conjecture by showing that the optimal two-bidder auction may not be ascending). Missing from these two papers, however, is a concrete sense of the 
ways in which this is a difficult problem. We provide this here.

There were several follow-up papers $[4,16,10]$, examining the extent to which simple mechanisms can achieve good approximations of Myerson's optimal mechanism, and motivated by Myerson's astonishing result that the optimal mechanism for the regular i.i.d. setting is simply a secondprice auction with reserve prices. For the most part, these are positive results yielding constant approximations by simple mechanisms in a variety of settings. Another line of work, is prior-free mechanism design, where the goal is to design mechanisms that achieve profits comparable to that of some well-behaved benchmark [13]. This direction became especially interesting after Hartline and Roughgarden developed a framework in [15] that is grounded in Bayesian optimal mechanism design allowing one to design mechanisms that simultaneously approximate all Bayesian optimal mechanisms. The intermediate approach of having bidders' valuations coming from a distribution that is nonetheless unknown to the auctioneer has also been considered [10]. Finally the important open problem of designing optimal multi-parameter mechanisms has been addressed for some distributions of preferences, such as additive valuations with budget constraints [2] and unit-demand settings [5] and connections of this problem to the algorithmic problem of optimal pricing have been made $[4,14]$; economists have also made some attempts to extend Myerson's results to multiitem auctions $[1,17]$.

In this paper we take a complexity-theoretic look at the general, correlated valuations case of Myerson's single-item auctions. We point out that the optimal auction design problem can be reduced essentially to a maximum independent set problem in a particular graph whose vertices are all possible tuples of valuations (an uncountable set, of course, in the continuous case). If the distribution is discrete, this is an ordinary graph-theoretic problem; no such combinatorial characterization had been known, and this had been the main difficulty in developing an algorithmic and complexitytheoretic understanding of the problem. For discrete distributions, this leads directly to an efficient algorithm in the case of two bidders, where the graph is bipartite, while in the case of three or more bidders NP-completeness (in fact, inapproximability) prevails. For continuous distributions, we get a duality characterization through a MongeKantorovich-like problem [12], and from this a fully polynomial approximation scheme for two bidders when the distribution is continuous enough and accessible through an oracle - plus, as an aside, a 2/3 approximation for three bidders, improving the previously best known approximation of [25]. We also point out two interesting consequences of our results for randomized auctions: The optimum deterministic auction is also optimum among all randomized truthful in expectation auctions for two bidders; and the optimal randomized truthful in expectation auction for any fixed number of three or more bidders, even though it may not be deterministic, can be computed in polynomial time.

Our results rest on a geometric characterization of optimal auctions. An important element of our proof are the so-called marginal profit contribution functions; these bear some similarities to Myerson's virtual valuation functions [20], the most important of them being that they both admit a marginal revenue interpretation in the spirit of the one in [3]. However, despite their similarities and their some- what common derivation, marginal profit contribution functions are different from Myerson's virtual valuations in a number of important ways: they only take positive values, they are not necessarily monotone and (unless the distribution happens to be a product one) they do not admit a natural interpretation as valuations in some modified domain. One important ingredient of Myerson's approach to the design of optimal auctions is an analytical maneuver he calls ironing; Myerson uses ironing to transform a potentially non-monotone allocation rule into a monotone one, without hurting revenue. Our approach circumvents ironing by restricting the space of mechanisms explored; we achieve that by imposing an additional technical condition which limits the design space into a subset of all mechanisms, but one which still contains all the optimal mechanisms.

The work most related to ours is that of Dobzinski, Fu and Kleinberg [11], which also studies the problem of designing the optimal auction for the correlated setting. They consider two models, one where the distribution is presented explicitly to the algorithm and one where the algorithm is allowed to ask conditional distribution queries to an oracle, and they give efficient algorithms for computing the optimal randomized truthful in expectation mechanism and a constant factor approximation thereof for each model respectively ${ }^{1}$ and for any number of bidders. They also consider deterministic mechanisms: they present an alternative derivation of our result, that the optimal two-bidder deterministic auction is computable in polynomial time and optimal among all truthful in expectation randomized mechanisms, and they also give a deterministic mechanism for $n$ bidders that guarantees a $3 / 5$ approximation to the revenue achievable by an optimal truthful in expectation mechanism; this last algorithm is the $k$-lookahead mechanism, first introduced and analyzed in [25], for which the authors present a clever improved analysis. Finally, they extend some of their results to get truthful in expectation mechanisms for the important multi-item case.

\section{PRELIMINARIES}

Imagine $n$ bidders seeking an indivisible good offered in auction. We assume every bidder has a private valuation $v_{i}$ for the item and that bidders' valuations are drawn from some distribution over $[0,1]^{n}$ whose density function we denote by $\phi(\mathbf{v})$. We consider both discrete and continuous $\phi$. In the continuous case, we assume that $\phi$ is Lipschitzcontinuous for some constant $\lambda$, and that we have oracle access to it (in such a way that, for example, it can be approximately integrated over nice regions). In both cases, we follow Myerson in making the analytically convenient assumption that $\phi$ is nowhere zero; that is, for every point in the unit square there is always a tiny probability that the valuations lie there (this enables the characterizations later in this section); any distribution is the limit of ones with this property.

We are interested in designing auctions that maximize the auctioneer's profit. Formally an auction consists of an allocation rule denoted by $x_{i}(\mathbf{v})$, the probability of bidder $i$ getting allocated the item, and a payment rule denoted by

\footnotetext{
${ }^{1}$ All the results about discrete distributions that are presented in our paper are for the explicit model; since we mostly focus on a small (constant) number of players and intractability, the explicit model is a realistic assumption and only strengthens our results.
} 
$p_{i}(\mathbf{v})$ which is the price paid by bidder $i$. In this paper we focus our attention on deterministic mechanisms so that $x_{i}(\mathbf{v}) \in\{0,1\}$. Our goal is to maximize the auctioneer's expected profit $\sum_{i=1}^{n} \mathbb{E}\left[\phi(\mathbf{v}) p_{i}(\mathbf{v})\right]$.

We want our auction to be ex-post incentive compatible (IC) and individually rational (IR), with no positive transfers (NPT). These notions are defineed as follows:

IC: $\forall i, v_{i}, v_{i}^{\prime}, v_{-i}: v_{i} x_{i}\left(v_{i}, v_{-i}\right)-p_{i}\left(v_{i}, v_{-i}\right) \geq v_{i} x_{i}\left(v_{i}^{\prime}, v_{-i}\right)-$ $p_{i}\left(v_{i}^{\prime}, v_{-i}\right)$

IR: $\forall i, v_{i}, v_{-i}: v_{i} x_{i}\left(v_{i}, v_{-i}\right)-p_{i}\left(v_{i}, v_{-i}\right) \geq 0$

$\mathrm{NPT}: \forall i, v_{i}, v_{-i}: p_{i}\left(v_{i}, v_{-i}\right) \geq 0$

We say that an allocation function $x_{i}\left(v_{i}, v_{-i}\right)$ is monotone if $v_{i} \geq v_{i}^{\prime}$ implies $x_{i}\left(v_{i}, v_{-i}\right) \geq x_{i}\left(v_{i}^{\prime}, v_{-i}\right)$ for all $i, v_{-i}$. For the case of deterministic mechanisms that we are interested in monotonicity implies that $x_{i}\left(v_{i}, v_{-i}\right)$ is a step-function. The threshold value of such a step-function allocation rule is set to be the minimum winning valuation for every player given the valuations of the other bidders: $t_{i}\left(v_{-i}\right)=\inf \left\{v_{i} \in\right.$ $\left.[0,1] \mid x_{i}\left(v_{i}, v_{-i}\right)=1\right\}$.

The following theorem provides a characterization of mechanisms for the setting we are interested in; a proof can be found in [22].

THEOREM 2.1. A deterministic mechanism satisfies IC, IR and NPT if and only if the following conditions hold.

1. $x_{i}\left(v_{i}, v_{-i}\right)$ is monotone.

2. For all $i, v_{i}, v_{-i}$ we have

$$
p_{i}\left(v_{i}, v_{-i}\right)= \begin{cases}t_{i}\left(v_{-i}\right) & \text { if } x_{i}\left(v_{i}, v_{-i}\right)=1 \\ 0 & \text { if } x_{i}\left(v_{i}, v_{-i}\right)=0 .\end{cases}
$$

In our last section we briefly discuss randomized mechanisms. In a randomized mechanism $x_{i}(\mathbf{v})$ is allowed to take any value in $[0,1]$ and it denotes the probability of bidder $i$ being allocated the item; analogously $p_{i}(\mathbf{v})$ denotes the expected payment of this agent. In this case a mechanism that satisfies the constraint IC above is called a randomized truthful in expectation mechanism, i.e. truthful for bidders that try to maximize their expected utility.

\section{GEOMETRY OF OPTIMAL AUCTIONS}

To simplify the exposition we are going to focus on the case of $n=2$; the geometric characterization (but not the Main Theorem in the next subsection) holds in general, with the appropriate, and rather straightforward, modifications and generalizations.

We start by noting that the allocation function can be described in terms of a partition of the unit square (the space of all possible valuation pairs) into three regions: In region $A$ bidder 1 gets the item, in region $B$ bidder 2 gets the item and in region $C$ neither gets the item. The shape of those regions is restricted by monotonicity as follows (see Figure 1): Region $A$ is rightward closed, meaning that $(x, y) \in A$ and $x^{\prime}>x$ implies $\left(x^{\prime}, y\right) \in A$, while region $B$ is, analogously, upward closed. These regions are captured by their boundaries: Region $A$ 's boundary is a function $\alpha:[0,1] \mapsto[0,1]$ where for all $y \in[0,1] \alpha(y)=\inf \{x:(x, y) \in A\}$, and similarly for region $B$ and its boundary $\beta(x)$.

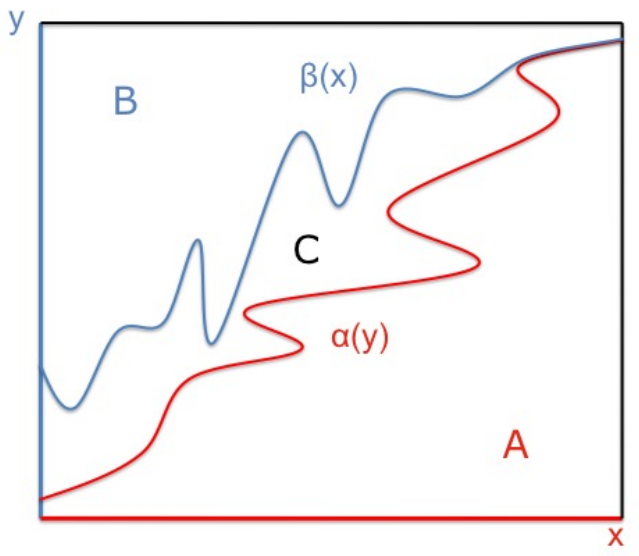

Figure 1: A pair of valid allocation rules

DEFINITION 3.1. A valid allocation pair $(\alpha, \beta)$ is a pair of functions from $[0,1]$ to itself satisfying the non-crossing property: for all $x, y \in[0,1]$ we have $y<\beta(\alpha(y))$ and $x<\alpha(\beta(x))$.

Notice how it is not necessary for the functions to be monotone; the monotonicity property of the allocation is ensured through the fact that $\alpha$ and $\beta$ are proper functions. The non-crossing property ensures that for any bid pair $(x, y)$ at most one bidder is allocated the item.

In our proof we will make extensive use of the following notion of marginal profit.

DeFinition 3.2. Let $f$ (resp. g) be the marginal profit contribution of a bid pair $(x, y)$ for player 1 (resp. 2) defined as:

$$
\begin{aligned}
& f(x, y)=-\frac{\partial}{\partial x}\left[\max _{x^{\prime} \geq x} x^{\prime} \cdot \int_{x^{\prime}}^{1} \phi(x, y) d x\right], \\
& g(x, y)=-\frac{\partial}{\partial y}\left[\max _{y^{\prime} \geq y} y^{\prime} \cdot \int_{y^{\prime}}^{1} \phi(x, y) d y\right]
\end{aligned}
$$

wherever the derivative is defined, and is extended to the full range by right continuity.

Intuitively, $f(x, y) d x d y$ is the added expected profit obtained from including the infinitesimal area $d x d y$ to $A$, that is, deciding to give the item to the first bidder if the valuations are $x, y$. These functions provide us an alternative way to express the objective of maximizing the expected profit:

LEMMA 3.3. Let $(\alpha, \beta)$ be a valid allocation pair that satisfies the following additional constraints, henceforth called the optimality constraints:

$$
\left.\begin{array}{l}
\alpha(y) \int_{\alpha(y)}^{1} \phi(x, y) d x=\max _{x^{\prime} \geq \alpha(y)} x^{\prime} \int_{x^{\prime}}^{1} \phi(x, y) d x \\
\beta(x) \int_{\beta(x)}^{1} \phi(x, y) d y=\max _{y^{\prime} \geq \beta(x)} y^{\prime} \int_{y^{\prime}}^{1} \phi(x, y) d y
\end{array}\right\}
$$

Then the expected profit of any auction with payments defined as in Theorem 2.1 is:

$$
\int_{0}^{1} \int_{\alpha(y)}^{1} f(x, y) d x d y+\int_{0}^{1} \int_{\beta(x)}^{1} g(x, y) d y d x
$$


Proof. Let $p_{1}(x, y), p_{2}(x, y)$ be the payment functions induced by the allocation rule $(a, b)$ according to Theorem 2.1. Then the expected profit of our auction is:

$$
\begin{aligned}
& \int_{0}^{1} \int_{0}^{1} p_{1}(x, y) \phi(x, y) d x d y+\int_{0}^{1} \int_{0}^{1} p_{2}(x, y) \phi(x, y) d x d y \\
= & \int_{0}^{1} \alpha(y) \int_{\alpha(y)}^{1} \phi(x, y) d x d y+\int_{0}^{1} \beta(x) \int_{\beta(x)}^{1} \phi(x, y) d y d x \\
= & \int_{0}^{1} \max _{x^{\prime} \geq \alpha(y)} x^{\prime} \int_{x^{\prime}}^{1} \phi(x, y) d x d y \\
& +\int_{0}^{1} \max _{y^{\prime} \geq \beta(x)} y^{\prime} \int_{y^{\prime}}^{1} \phi(x, y) d y d x \\
= & \int_{0}^{1} \int_{\alpha(y)}^{1} f(x, y) d x d y+\int_{0}^{1} \int_{\beta(x)}^{1} g(x, y) d y d x
\end{aligned}
$$

It is easy to verify that condition (1) is not a loss of generality, in the sense that in order to find the optimal auction we can restrict our attention to allocation rules that satisfy it:

Lemma 3.4. For any valid allocation pair $(\alpha, \beta)$ there exists another valid pair $\left(\alpha^{\prime}, \beta^{\prime}\right)$ such that Profit $\left(\alpha^{\prime}, \beta^{\prime}, \phi\right) \geq$ Profit $(\alpha, \beta, \phi)$ and condition (1) holds for $\left(\alpha^{\prime}, \beta^{\prime}\right)$.

Proof. The idea is to gradually turn $(\alpha, \beta)$ into $\left(\alpha^{\prime}, \beta^{\prime}\right)$. Let for example point $(x, y)$ violate the condition for player 1, i.e. $\quad \alpha(y) \int_{\alpha(y)}^{1} \phi(x, y) d x<\max _{x^{\prime} \geq \alpha(y)} x^{\prime} \int_{x^{\prime}}^{1} \phi(x, y) d x$. We can get rid of this violation by simply increasing $\alpha(y)$ to $\alpha^{\prime}(y)=\arg \max _{x^{\prime} \geq \alpha(y)} x^{\prime} \int_{x^{\prime}}^{1} \phi(x, y) d x .^{2}$ It is easy to verify that the resulting allocation pair is still valid: both the monotonicity and the non-crossing property are satisfied. Moreover using Lemma 3.3 we can verify that the profit can only increase. We then repeat until we get rid of all violations.

We denote by $\mathcal{A B}$ the set of all valid allocations $(\alpha, \beta)$ satisfying condition (1). The problem of finding the optimal auction can now be restated as the following variational calculus-type problem:

\section{Definition 3.5. [Problem A]}

$$
\sup _{(\alpha, \beta) \in \mathcal{A B}}\left\{\int_{0}^{1} \int_{\alpha(y)}^{1} f(x, y) d x d y+\int_{0}^{1} \int_{\beta(x)}^{1} g(x, y) d y d x\right\}
$$

\subsection{The Main Theorem}

Our main result is a duality theorem. We show that the maximization Problem A is equivalent to a certain massmoving problem (reminiscent in some aspects of the classic Monge-Kantorovich [12] mass-transfer problem), namely the following:

\footnotetext{
${ }^{2}$ In case there are more than one points in $\arg \{\cdot\}$, we just arbitrarily pick one, say the rightmost.
}

Definition 3.6. [Problem B]

$$
\begin{aligned}
& \inf _{\gamma}\left\{\int_{0}^{1} \int_{0}^{1} \int_{0}^{y_{1}} \int_{x_{1}}^{1} \gamma\left(x_{1}, y_{1}, x_{2}, y_{2}\right) d x_{2} d y_{2} d x_{1} d y_{1}\right\} \\
& \text { s.t. } \forall\left(x_{1}, y_{1}\right) \in[0,1]^{2}, \forall\left(x_{2}, y_{2}\right) \in[0,1]^{2}: \\
& \int_{0}^{y_{1}} \int_{x_{1}}^{1} \gamma\left(x_{1}, y_{1}, x_{2}, y_{2}\right) d x_{2} d y_{2} \geq f\left(x_{1}, y_{1}\right) \\
& \int_{y_{2}}^{1} \int_{0}^{x_{2}} \gamma\left(x_{1}, y_{1}, x_{2}, y_{2}\right) d x_{1} d y_{1} \geq g\left(x_{2}, y_{2}\right) \\
& \gamma\left(x_{1}, y_{1}, x_{2}, y_{2}\right) \geq 0
\end{aligned}
$$

Let us denote by $\Gamma$ the set of all functions $\gamma:[0,1]^{4} \mapsto \Re$ satisfying the above constraints.

One intuitive interpretation of Problem B is this: We are given two landscapes in the unit square, captured by two functions $f, g:[0,1]^{2} \mapsto \Re$. We are seeking a plan for transforming landscape $f$ to landscape $g$, where the following operations are allowed:

- take material away from any point $(x, y)$;

- add material to any point $(x, y)$;

- transfer material from any point to any other point in the southeast direction (if some material is not moved, we think of it as having moved in the southeast direction zero distance).

We want the plan in which the total amount of material moved (irrespective of distance moved, here is where this problem differs significantly from Monge-Kantorovitch) is minimized. Our main theorem is that this problem coincides, at optimality, with the optimal auction:

THEOREM 3.7. [Main]

$$
\begin{aligned}
& \sup _{(\alpha, \beta) \in \mathcal{A B}}\left\{\int_{0}^{1} \int_{\alpha(y)}^{1} f(x, y) d x d y+\int_{0}^{1} \int_{\beta(x)}^{1} g(x, y) d y d x\right\} \\
& =\inf _{\gamma \in \Gamma}\left\{\int_{0}^{1} \int_{0}^{1} \int_{0}^{y_{1}} \int_{x_{1}}^{1} \gamma\left(x_{1}, y_{1}, x_{2}, y_{2}\right) d x_{2} d y_{2} d x_{1} d y_{1}\right\}
\end{aligned}
$$

\subsection{Proof of the Main Theorem}

\subsubsection{General Plan}

The proof of the Theorem is by discretizing the unit square, proving a duality result for the discrete version, establishing upper bounds for the discretization error, and taking the limit for finer and finer discretization. We shall first define two auxiliary problems that will be useful in our proof.

\subsubsection{Discretization}

We start by discretizing the continuous functions $f$ and $g$ defined on $[0,1] \times[0,1]$ by two discrete functions $f_{d}$ and $g_{d}$ defined on the $[n] \times[n]$ grid, where $n$ is an integer greater than one and $[\mathrm{n}]=\{0,1, \ldots, \mathrm{n}-1\}$; we denote $\frac{1}{n}$ by $\epsilon$. We subdivide the $[0,1] \times[0,1]$ square into $\epsilon \times \epsilon$ little squares; we are implicitly mapping a little square with southwest coordinate $(x, y)$ to the grid point $n \cdot(x, y)$.

The discrete functions are now obtained by assigning to each point in the grid the aggregate mass of its corresponding square on the plane. 
$f_{d}(i, j)=\int_{\epsilon i}^{\epsilon(i+1)} \int_{\epsilon j}^{\epsilon(j+1)} f(x, y) d y d x$, for $i, j=0, \ldots, n-1$

and

$$
g_{d}(i, j)=\int_{\epsilon i}^{\epsilon(i+1)} \int_{\epsilon j}^{\epsilon(j+1)} g(x, y) d y d x \text { for } i, j=0, \ldots, n-1 .
$$

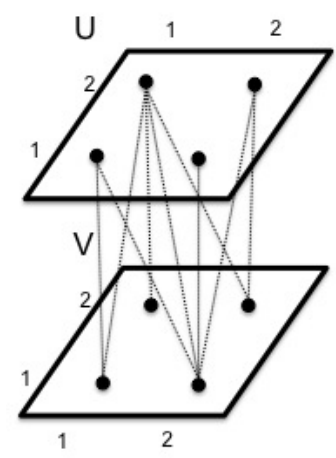

Figure 2: The graph consisting of two grids, one for each player, for $n=2$.

\subsubsection{The Graph}

We next create a weighted bipartite graph $G=(U, V, E)$ as follows: $U=V=[n] \times[n]$. Each vertex $u=(i, j)$ of $U$ has a weight equal to $w_{u}=f_{d}(i, j)+2 \epsilon^{3} \lambda$, and similarly each vertex $v=\left(i^{\prime}, j^{\prime}\right)$ of $V$ has a weight $w_{v}=g_{d}\left(i^{\prime}, j^{\prime}\right)+2 \epsilon^{3} \lambda$. A pair $\left((i, j),\left(i^{\prime}, j^{\prime}\right)\right)$ is in $E$ if and only if $i \leq i^{\prime}$ and $j \geq j^{\prime}$, that is, if grid point $v$ is in the Southeast direction from grid point $u$ (see Figure 2 for an example with $n=2$ ).

Consider now the following problem:

Definition 3.8. [Problem C: Maximum Weight InDEPENDENT SET]

Given the weighted bipartite graph $G=(U, V, E)$ above,

$$
\begin{array}{cl}
\max _{\left\{x_{u} \in\{0,1\}\right\}} & \sum_{u \in U \cup V} x_{u} w_{u} \\
\text { s.t. } & x_{u}+x_{v} \leq 1, \forall(u, v) \in E
\end{array}
$$

The dual of the above problem is the following:

Definition 3.9. [Problem D: Minimum Cost TransSHIPMENT]

Given the weighted bipartite graph $G=(U, V, E)$ above,

$$
\begin{array}{cl}
\min _{\left\{y_{u v} \in \Re\right\}} & \sum_{(u, v) \in E} y_{u v} \\
\text { s.t. } & \sum_{v:(u, v) \in E} y_{u v} \geq w_{u}, \forall u \in U \\
& \sum_{u:(u, v) \in E} y_{u v} \geq w_{v}, \forall v \in V \\
& y_{u v} \geq 0, \forall(u, v) \in E
\end{array}
$$

\subsubsection{The Inequalities}

The crux of the proof is a sequence of results relating the various solutions and optimum solutions of these four problems. In what follows we use $S O L(\cdot)$ to denote the cost of any feasible solution of a problem among (A), (B), (C), and $(\mathrm{D})$ defined above, and $O P T(\cdot)$ to denote the cost of the optimum solution of a problem (sometimes $S O L$ and $O P T$ also denotes the actual solutions).

We start off with a lemma that establishes that functions $f$ and $g$ are Lipschitz-continuous.

LEMMA 3.10. If the probability distribution $\phi$ is Lipschitzcontinuous, then the marginal profit contribution functions $f$ and $g$ are also Lipschitz-continuous.

We next prove some inequalities from which the main theorem follows; the first one establishes a form of weak duality between problems $A$ and $B$, while the next two show that the discretization error is small. All proofs can be found in Appendix A.

LEMMA 3.11. For any feasible solutions of $A$ and $B$ we have: $\operatorname{SOL}(A) \leq \operatorname{SOL}(B)$.

Lemma 3.12. For the optimal solutions of $A$ and $C$ we have: $\operatorname{OPT}(A) \geq O P T(C)-4 \epsilon \lambda$.

Lemma 3.13. For the optimal solutions of $B$ and $D$ we have: $\operatorname{OPT}(D) \geq O P T(B)$.

Proof. [Main Theorem] First notice that, by strong duality and since the constraint matrix is totally unimodular, we have that $O P T(C)=O P T(D)$; combining this with Lemmas 3.12 and 3.13 we get $O P T(A) \geq O P T(B)-\Theta(\epsilon)$. From Lemma 3.11 we get $O P T(A) \leq O P T(B)$. By having $\epsilon \rightarrow 0$ we get the result.

\subsection{An optimal mechanism for two bidders}

It is immediate that the proof of the Main Theorem suggests a polynomial-time algorithm for the two bidders case when the distribution is discrete (solving the maximum independent set problem through min-cost flow). For the continuous case it yields an FPTAS, that is, a mechanism that approximates the optimal profit within $\epsilon$ (additive error) and runs in time polynomial in $\Theta\left(1 / \epsilon^{2}\right)$. The algorithm is the following:

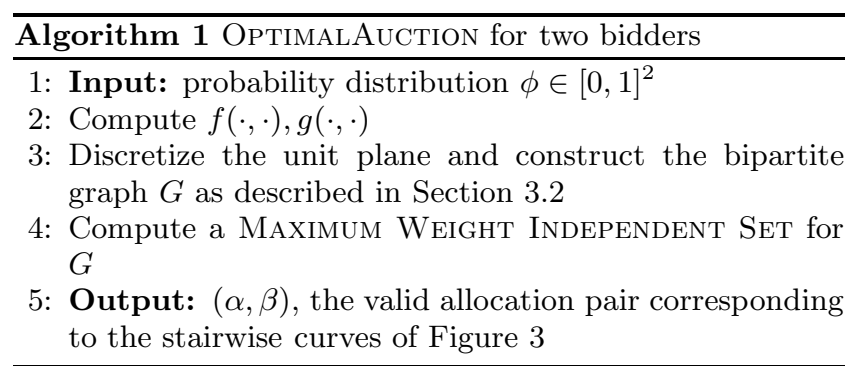

Notice that the pair of functions $(\alpha, \beta)$ returned may well not satisfy condition (1) and may consequently not constitute a feasible solution to Problem A. Therefore, to compare the profit of our auction to that of the optimal auction, which is approximated through Problem C, we need to show one additional property of the optimal boundary curves $(\alpha, \beta)$, namely that they have bounded slope.

In the next lemma we prove the more general statement that any boundaries of a sub-region of the unit square with $f(x, y)=0$ (or $g(x, y)=0)$ must have bounded slope. Its proof can be found in Appendix A.5. 
LEMma 3.14. The boundary between regions with $f(x, y)=$ 0 and $f(x, y) \neq 0$ has finite (bounded) slope. The same holds for $g(x, y)$.

The following theorem establishes that our algorithm has the desired properties; its proof is deferred to Appendix A.6.

THEOREM 3.15. Algorithm 1 is a truthful mechanism that approximates the optimal profit within $\epsilon$ additive error and runs in time polynomial in $1 / \epsilon$.

\section{HARDNESS OF APPROXIMATING THE OPTIMAL AUCTION}

We show that for 3 bidders the problem of designing an approximately optimal (deterministic) auction becomes NPhard for some threshold. We show it for discrete distributions, but the continuous case follows easily.

Definition 4.1. Let 3OptimalAuctionDesign be the following problem: given a 3-dimensional matrix that represents a discrete probability distribution $\phi(x, y, z)$ with finite support $S$, design the optimal, ex-post truthful and IR deterministic auction and output its 3-dimensional allocation matrix.

Our characterization of Section 3, generalized to three dimensions, implies that 3OptimalAUCTIONDESIGN is equivalent to the Maximum Weight IndePendent Set problem for a particular type of tripartite graphs: the nodes of every player are a $|S| \times|S| \times|S|$ grid, with weights equal to the marginal profit contribution ${ }^{3}$ of the corresponding player at each point, and the edges are defined in complete analogy with the 2-bidder case.

In what follows, instead of representing those graphs in terms of nodes and edges, we use the following equivalent, more convenient representation. Consider a node $(x, y, z)$ on the 3-dimensional grid such that $f(x, y, z)>0$; this node is the starting point of what we shall henceforth be calling a segment: an interval (sequence of points) starting at node $(x, y, z)$ and including all nodes $\left(x^{\prime}, y, z\right)$ with $x^{\prime} \geq x$. The weight of this segment is set to be $\sum_{x^{\prime} \geq x} f\left(x^{\prime}, y, z\right)$. We define segments across the other dimensions analogously. Based on this construction, we can see that every instance of 3OptimalAuctionDEsign gives rise to an instance of the following problem:

Definition 4.2 (3SEgments). We are given as input a discrete probability distribution $\phi(x, y, z)$ which induces a set of segments on the 3-dimensional grid as described above; we are asked to find a subset of non-intersecting segments with maximum sum of weights.

Lemma 4.3. The problems 3OptimalAuctionDesign, MaXIMUM WEIGHT INDEPENDENT SET on the induced tripartite graph and 3SEGMENTS are all equivalent via approximationpreserving reductions.

\footnotetext{
${ }^{3}$ The discrete analogue of the marginal profit contribution functions (Definition 3.2) for -sayplayer 1 can be easily seen to be $f(x, y, z)=$ $\max \left\{x \cdot \sum_{x^{\prime} \geq x} \phi\left(x^{\prime}, y, z\right)-\sum_{x^{\prime}>x} f\left(x^{\prime}, y, z\right), 0\right\}, \quad$ with $f(1, y, z)=\phi(1, y, z)$.
}

Proof. Equivalence of 3OptimalAuctionDesign and Maximum Weight Independent Set on the induced tripartite graph follows from the proof of the Main Theorem.

To see that 3SEGMENTs is also equivalent with the other two, first notice that the monotonicity constraint is readily satisfied since segments are defined to be rightward (resp. upward etc) closed. The non-crossing property is ensured by the non-crossing of the segments selected in the solution, and condition (1) is satisfied because we only introduce segments for nodes with non-zero weight. Finally it is easy to verify that a 3SEGMENTS solution can be transformed into a valid auction for the same input distribution $\phi$ with expected profit equal to the sum of the segments' weights.

\subsection{The reduction}

It suffices to show that 3SEGMENTS is NP-hard to approximate for some constant; we do that by reducing from 3CATSAT, a special case of 3SAT:

DeFinition 4.4. Let 3CATSAT be the 3SAT problem with the input formula restricted to be of the following form. We have three categories of variables $\left\{x_{i}\right\}_{i=1 \ldots n_{x}},\left\{y_{i}\right\}_{i=1 \ldots n_{y}}$ and $\left\{z_{i}\right\}_{i=1 \ldots n_{z}}$, i.e. a total of $n=n_{x}+n_{y}+n_{z}$ variables, and $m$ clauses of the following form: every clause has at most one literal from every type (e.g. $\overline{x_{2}} \vee \overline{y_{3}} \vee z_{1}, \overline{x_{5}} \vee z_{7}$ ).

The proof for the following lemma is easy and is deferred to the full version of the paper.

LEMMA 4.5. 3CATSAT is NP-hard to approximate better than $103 / 104$.

We shall only give here some intuition about the reduction. The instance of 3SEGMENTS we create has three types of segments: literal-segments, clause-segments and scaffoldsegments.

- Literal-segments are used to model truth assignments on variables; they ensure that every variable is assigned exactly one of the two possible truth-values and that this assignment is consistent across all appearances of literals of this particular variable.

- Clause-segments model the truth assignment to literals of a particular clause; we create one such segment for every literal that appears in a clause and we make them intersect with the literal-segments of the negations of those literals; the idea is that if the clause is satisfied we will be able to pick at least one clause-segment per clause because the corresponding literal-segment will not be picked. Moreover, we cannot pick more than one clause-segments per clause, since they will all intersect with each other.

- Scaffold-segments (or points) ensure that we only have segments in the desired directions and nothing more.

Our main result for this section is then the following:

TheOREM 4.6. It is NP-hard to approximate 3SEGMENTS better than $0.03 \%$.

The details of the construction as well as the proof of the above theorem can be found in the full version of the paper. 


\section{OPTIMAL RANDOMIZED AUCTIONS}

Even though in this paper we focused on deterministic mechanisms, our geometric characterization has interesting consequences for randomized mechanisms. For simplicity, in stating the related results we assume discrete distributions, although similar results hold for the continuous case, as before modulo a discretization error that can be made arbitrarily small. Remember that for the discrete case the optimal (deterministic) mechanism immediately follows from solving the integer program of problem $\mathrm{C}$ in the previous section, i.e. computing a maximum weight independent set in the corresponding $n$-partite graph.

We first point out that the linear programming relaxation of this integer program corresponds to computing the optimal randomized truthful in expectation mechanism. For two players, where the graph is bipartite and the integer program is totally unimodular, the optimum integer solution is also the optimum of the relaxed linear program. Therefore, for two bidders, the program of problem $\mathrm{C}$ computes a deterministic mechanism that is optimal among all randomized mechanisms: this is reminiscent of Myerson's original result, where the deterministic mechanism obtained is optimal for the (larger) class of Bayesian truthful randomized mechanisms [20]. For a constant number of three or more bidders, the generalization of our geometric characterization (which we have omitted in this version of our paper) yields polynomial-time algorithms for computing the optimal randomized auction, in sharp contrast with the intractability of computing the optimal deterministic auction, even for three bidders.

ThEOREM 5.1. For the discrete case of the single-item auction problem:

1. The optimal deterministic mechanism for two bidders is optimal among all randomized truthful in expectation mechanisms.

2. For a constant number of three or more bidders, randomized mechanisms may achieve more revenue than deterministic ones. However, the optimal (truthful in expectation) randomized mechanism can be computed in polynomial time.

Proof. Consider the linear program for problem $\mathrm{C}$ given in the previous section. As we have seen, its optimum solution is integer, and corresponds to the optimum auction. It is also easy to see that the set of fractional feasible solutions comprises the set of all randomized auctions, with $x_{u}$ standing for the probability that, if the valuations are as in the apex of segment $u$, the item will go to the bidder corresponding to the direction of segment $u$. Since this LP is totally unimodular, it follows that the integer solution is optimum. This means precisely that, for two bidders, the optimum deterministic auction is also optimum among probabilistic auctions, establishing claim (1).

To show (2), for each point $p$ in the support, and for each bidder $i$, we have a random variable $x_{i}^{p}$ standing for the probability that bidder $i$ will be allocated the item if $p$ prevails. The constraints are the same as in the two-bidder case: we consider the line segment $S_{i}^{p}$ starting at $p$ and extending maximally parallel to the $i$-th axis as associated with variable $x_{i}^{p}$. At every point at which two or more such segments intersect, we demand that the corresponding variables add up to one or less. The resulting LP captures the optimum randomized auction design problem for three or more bidders, except that the underlying structure is no longer a bipartite graph but a tripartite (or more) hypergraph (and hence the LP may no longer be totally unimodular). Solving this LP will yield the optimum randomized auction.

For a large number of bidders, the size of this linear program may become exponentially large; for an alternative linear program that computes the optimal randomized auction for any number of bidders, when the distribution is given explicitly, the reader is referred to [11].

\section{CONCLUSION AND OPEN PROBLEMS}

We have explored the territory of optimal Bayesian singleitem auction design beyond Myerson's landmark result. We have shown that for two bidders the optimal auction can be calculated via a reduction to an independent set problem, while for three or more bidders it becomes intractable. This is the first non trivial optimal auction beyond Myerson's paper, and the first complexity result in this setting as well. One immediate question is to come up with an interpretation for our two-bidder optimal auction that connects it to Myerson's which it partly generalizes.

Another important question is to close the gap between the best approximation algorithm known for the optimal auction problem (.50) and the inapproximability bound (currently about .9997). We believe that progress there is attainable. Interestingly, our work implies an approximation of $2 / n$ for $n$ players: before having the bidders announce their bids, the auctioneer looks at their joint distribution and privately runs the optimal auction for all possible pairs of players. Since solving for the optimal auction is nothing but a maximum weight independent set problem on the corresponding graph, it is easy to prove that the profit of the best of those $\left(\begin{array}{c}n \\ 2\end{array}\right)$ auctions is at least $2 / n$ of the overall profit. The auctioneer then rejects a priori all but the bidders who were part of the most profitable two-bidder auction and then runs it. The overall auction is obviously truthful as long as bidders are rejected before even submitting their bids. For 3 bidders this gives an approximation ratio of $2 / 3$, improving over Ronen's auction, but for $n \geq 4$ the approximation ratio drops below $1 / 2$. Another direction is to consider general single-dimensional feasibility settings, such as downward closed settings and matroids [16].

Of course, it would be interesting to see if an analogous approach could yield some insight and new results for the very important and challenging problem of designing optimal multi-dimensional mechanisms.

\section{ACKNOWLEDGMENTS}

We thank Adam Szeidl, Jason Hartline and Tim Roughgarden for interesting feedback and the anonymous STOC reviewers for their useful comments. $\mathrm{CP}$ wants to thank to Benny Moldovanu for encouraging him and inspiring him to look at this problem. We are also grateful to Shahar Dobzinski, Hu Fu and Bobby Kleinberg for comments on Section 5 and for sharing with us an early manuscript of their work [11].

\section{REFERENCES}

[1] M. Armstrong. Optimal multi-object auctions. Review of Economic Studies, 67(3):455-81, July 2000. 
[2] S. Bhattacharya, G. Goel, S. Gollapudi, and K. Munagala. Budget constrained auctions with heterogeneous items. In STOC, pages 379-388, 2010.

[3] J. Bulow and J. Roberts. The simple economics of optimal auctions. Journal of Political Economy, 97(5):1060-90, October 1989.

[4] S. Chawla, J. D. Hartline, and R. D. Kleinberg. Algorithmic pricing via virtual valuations. In $A C M$ Conference on Electronic Commerce, pages 243-251, 2007.

[5] S. Chawla, J. D. Hartline, D. L. Malec, and B. Sivan. Multi-parameter mechanism design and sequential posted pricing. In STOC, pages 311-320, 2010.

[6] X. Chen and X. Deng. Settling the complexity of two-player nash equilibrium. In FOCS, pages 261-272, 2006.

[7] J. Cremer and R. P. McLean. Optimal selling strategies under uncertainty for a discriminating monopolist when demands are interdependent. Econometrica, 53(2):345-61, March 1985.

[8] J. Cremer and R. P. McLean. Full extraction of the surplus in bayesian and dominant strategy auctions. Econometrica, 56(6):1247-57, November 1988.

[9] C. Daskalakis, P. W. Goldberg, and C. H. Papadimitriou. The complexity of computing a nash equilibrium. In $S T O C$, pages 71-78, 2006.

[10] P. Dhangwatnotai, T. Roughgarden, and Q. Yan. Revenue maximization with a single sample. In $A C M$ Conference on Electronic Commerce, pages 129-138, 2010.

[11] S. Dobzinski, H. Fu, and R. Kleinberg. Optimal auctions with correlated bidders are easy. In STOC, 2011.

[12] L. C. Evans. Partial differential equations and monge-kantorovich mass transfer (surveypaper. In Current Developments in Mathematics, 1997, International Press, 1999.

[13] A. V. Goldberg, J. D. Hartline, A. R. Karlin, A. Wright, and M. Saks. Competitive auctions. In Games and Economic Behavior, pages 72-81, 2002.

[14] V. Guruswami, J. D. Hartline, A. R. Karlin, D. Kempe, C. Kenyon, and F. McSherry. On profit-maximizing envy-free pricing. In $S O D A$, pages 1164-1173, 2005.

[15] J. D. Hartline and T. Roughgarden. Optimal mechanism design and money burning. In STOC, pages 75-84, 2008.

[16] J. D. Hartline and T. Roughgarden. Simple versus optimal mechanisms. In ACM Conference on Electronic Commerce, pages 225-234, 2009.

[17] P. Jehiel, M. Meyer-ter Vehn, and B. Moldovanu. Mixed bundling auctions. Journal of Economic Theory, 134(1):494-512, May 2007.

[18] E. Koutsoupias and C. H. Papadimitriou. Worst-case equilibria. In STACS, pages 404-413, 1999.

[19] R. P. McAfee and P. J. Reny. Correlated information and mechanism design. Econometrica, 60(2):395-421, March 1992.

[20] R. B. Myerson. Optimal auction design. Discussion Papers 362, Northwestern University, Center for
Mathematical Studies in Economics and Management Science, Dec. 1978.

[21] N. Nisan and A. Ronen. Algorithmic mechanism design (extended abstract). In $S T O C$, pages 129-140, 1999.

[22] N. Nisan, T. Roughgarden, É. Tardos, and V. V. Vazirani. Algorithmic Game Theory. Cambridge University Press, New York, NY, USA, 2007.

[23] C. H. Papadimitriou. Algorithms, games, and the internet. In $I C A L P$, pages $1-3,2001$.

[24] C. H. Papadimitriou, M. Schapira, and Y. Singer. On the hardness of being truthful. In FOCS, pages 250-259, 2008.

[25] A. Ronen. On approximating optimal auctions. In ACM Conference on Electronic Commerce, pages 11-17, 2001.

[26] A. Ronen and A. Saberi. On the hardness of optimal auctions. In FOCS, pages 396-405, 2002.

[27] T. Roughgarden. The price of anarchy is independent of the network topology. J. Comput. Syst. Sci., 67(2):341-364, 2003.

[28] T. Roughgarden and É. Tardos. How bad is selfish routing? J. ACM, 49(2):236-259, 2002.

[29] W. Vickrey. Counterspeculation, auctions, and competitive sealed tenders. The Journal of Finance, 16(1):8-37, 1961.

\section{APPENDIX}

\section{A. MISSING PROOFS}

\section{A.1 Proof of Lemma 3.10}

We only show the result for $f$ and the proof for $g$ is identical. To show Lipschitz-continuity along the $x$-axis it suffices to note that $f(x, y)=\max \left\{0, x \phi(x, y)-\int_{x}^{1} \phi(x, y) d x\right\}$ and remember that $\phi$ is Lipschitz-continuous. For the Lipschitzcontinuity along the $y$-axis, first notice that the function $x \phi(x, y)-\int_{x}^{1} \phi(x, y) d x$ is Lipschitz-continuous along the $y$ axis as well; by taking the maximum of this function and zero $|f(x, y)-f(x, y \pm \epsilon)|$ can only decrease.

\section{A.2 Proof of Lemma 3.11}

$$
\begin{aligned}
& \operatorname{SOL}(A) \\
= & \int_{0}^{1} \int_{\alpha\left(y_{1}\right)}^{1} f\left(x_{1}, y_{1}\right) d x_{1} d y_{1}+\int_{0}^{1} \int_{\beta\left(x_{2}\right)}^{1} g\left(x_{2}, y_{2}\right) d y_{2} d x_{2} \\
\leq & \int_{0}^{1} \int_{\alpha\left(y_{1}\right)}^{1} \int_{0}^{y_{1}} \int_{x_{1}}^{1} \gamma\left(x_{1}, y_{1}, x_{2}, y_{2}\right) d x_{2} d y_{2} d x_{1} d y_{1} \\
& +\int_{0}^{1} \int_{\beta\left(x_{2}\right)}^{1} \int_{y_{2}}^{1} \int_{0}^{x_{2}} \gamma\left(x_{1}, y_{1}, x_{2}, y_{2}\right) d x_{1} d y_{1} d y_{2} d x_{2} \\
\leq & \int_{0}^{1} \int_{\alpha\left(y_{1}\right)}^{1} \int_{0}^{y_{1}} \int_{x_{1}}^{1} \gamma\left(x_{1}, y_{1}, x_{2}, y_{2}\right) d x_{2} d y_{2} d x_{1} d y_{1} \\
& +\int_{0}^{1} \int_{0}^{\alpha\left(y_{2}\right)} \int_{y_{2}}^{1} \int_{0}^{x_{2}} \gamma\left(x_{1}, y_{1}, x_{2}, y_{2}\right) d x_{1} d y_{1} d x_{2} d y_{2} \\
\leq & \int_{0}^{1} \int_{\alpha\left(y_{1}\right)}^{1} \int_{0}^{y_{1}} \int_{x_{1}}^{1} \gamma\left(x_{1}, y_{1}, x_{2}, y_{2}\right) d x_{2} d y_{2} d x_{1} d y_{1}
\end{aligned}
$$




$$
\begin{aligned}
& +\int_{0}^{1} \int_{0}^{\alpha\left(y_{2}\right)} \int_{0}^{y_{2}} \int_{x_{2}}^{1} \gamma\left(x_{2}, y_{2}, x_{1}, y_{1}\right) d x_{1} d y_{1} d x_{2} d y_{2} \\
= & \int_{0}^{1} \int_{\alpha\left(y_{1}\right)}^{1} \int_{0}^{y_{1}} \int_{x_{1}}^{1} \gamma\left(x_{1}, y_{1}, x_{2}, y_{2}\right) d x_{2} d y_{2} d x_{1} d y_{1} \\
& +\int_{0}^{1} \int_{0}^{\alpha\left(y_{1}\right)} \int_{0}^{y_{1}} \int_{x_{1}}^{1} \gamma\left(x_{1}, y_{1}, x_{2}, y_{2}\right) d x_{2} d y_{2} d x_{1} d y_{1} \\
= & \int_{0}^{1} \int_{0}^{1} \int_{0}^{y_{1}} \int_{x_{1}}^{1} \gamma\left(x_{1}, y_{1}, x_{2}, y_{2}\right) d x_{2} d y_{2} d x_{1} d y_{1} \\
= & \operatorname{SOL}(B)
\end{aligned}
$$

where in the first inequality we used the inequality constraints on $\gamma$ and in the second inequality we rearranged the order of integration using the fact that $(\alpha, \beta)$ break the plane into disjoint regions (non-crossing property) and the non-negativity of $\gamma$; in the third inequality we again rearranged the order of integration, by using the fact that (because curve $\alpha$ is a proper function) for every point $\left(x_{2}, y_{2}\right)$, all points $\left(x_{1}, y_{1}\right)$ lying in its northwest orthant are also included in the (outer) integration as $\left(x_{2}, y_{2}\right)$ points; we also used the non-negativity of $\gamma$. In the next equality we simply renamed variables.

\section{A.3 Proof of Lemma 3.12}

Consider a solution of problem $C$; this has the shape of Figure 3, which means that the borders of those regions consist a valid allocation pair. Indeed, the edges of graph $G$ model the constraint of allocating the item only to one player (non-crossing property), for all possible bid pairs. To see why the monotonicity property is satisfied consider two points $(i, j)$ and $\left(i^{\prime}, j\right)$ on player 1's grid, where $i^{\prime}>i$. Notice that point $(i, j)$ of player 1 's grid is connected to strictly more points on player 2's grid than $\left(i^{\prime}, j\right)$. Hence, if the maximum weight independent set includes point $(i, j)$ in the grid of player 1 , it should also include $\left(i^{\prime}, j\right)$ for all values $i^{\prime}>i$ (except for the case of zero-weight nodes; to get around this we can just restrict attention to maximum cardinality solutions).

This gives us two stairwise curves which -although being a valid allocation pair- fail to satisfy Condition 1, and hence are not a feasible solution for problem $A$. To turn them into feasible solutions, we can simply follow the same procedure as in Lemma 3.4 and come up with a feasible solution $S O L(A)$ for problem $A$.

The cost of this solution can be easily seen to be:

$$
\begin{aligned}
& \sum_{u \in U} x_{u} f_{d}(u)+\sum_{v \in V} x_{v} g_{d}(v) \\
\geq & \sum_{u \in U} x_{u} w_{u}+\sum_{v \in V} x_{v} w_{v}-\frac{2}{\epsilon^{2}} \cdot 2 \epsilon^{3} \lambda \\
= & O P T(C)-4 \epsilon \lambda
\end{aligned}
$$

and the theorem follows.

\section{A.4 Proof of Lemma 3.13}

Given a feasible solution for problem $D$, we come up with a feasible solution of the same cost for problem $B$. We start by defining $\gamma\left(x_{1}, y_{1}, x_{2}, y_{2}\right)$, for any pair of points $\left(x_{1}, y_{1}\right),\left(x_{2}, y_{2}\right)$, with $\left(x_{2}, y_{2}\right)$ lying in the southeast orthant

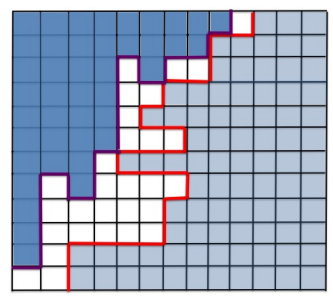

Figure 3: A feasible solution for problem $C$ and the stairwise curves $\alpha, \beta$.

of $\left(x_{1}, y_{1}\right)$ (i.e. $\left.x_{1} \leq x_{2}, y_{1} \geq y_{2}\right)$ to be:

$\gamma\left(x_{1}, y_{1}, x_{2}, y_{2}\right)=\left\{\begin{array}{l}y_{u, v} / \epsilon^{4}, \\ \quad \text { if } x_{2} \geq\left\lceil\frac{x_{1}}{\epsilon}\right\rceil \cdot \epsilon, y_{2} \leq\left\lfloor\frac{y_{1}}{\epsilon}\right\rfloor \cdot \epsilon ; \\ \\ y_{u, v} / \epsilon^{3}\left(y_{1}-\left\lfloor\frac{y_{1}}{\epsilon}\right\rfloor \cdot \epsilon\right), \\ \quad \text { if } x_{2} \geq\left\lceil\frac{x_{1}}{\epsilon}\right\rceil \cdot \epsilon, y_{1} \geq y_{2} \geq\left\lfloor\frac{y_{1}}{\epsilon}\right\rfloor \cdot \epsilon ; \\ y_{u, v} / \epsilon^{3}\left(\left\lceil\frac{x_{1}}{\epsilon}\right\rceil \cdot \epsilon-x_{1}\right), \\ \quad \text { if } x_{1} \leq x_{2} \leq\left\lceil\frac{x_{1}}{\epsilon}\right\rceil \cdot \epsilon, y_{2} \leq\left\lfloor\frac{y_{1}}{\epsilon}\right\rfloor \cdot \epsilon ; \\ y_{u, v} / \epsilon^{2}\left(y_{1}-\left\lfloor\frac{y_{1}}{\epsilon}\right\rfloor \cdot \epsilon\right)\left(\left\lceil\frac{x_{1}}{\epsilon}\right\rceil \cdot \epsilon-x_{1}\right), \\ \text { if } x_{1} \leq x_{2} \leq\left\lceil\frac{x_{1}}{\epsilon}\right\rceil \cdot \epsilon, y_{1} \geq y_{2} \geq\left\lfloor\frac{y_{1}}{\epsilon}\right\rfloor \cdot \epsilon ;\end{array}\right.$

where $u \in U$ (resp. $v \in V)$ is the grid point $\left(\left\lfloor\frac{x_{1}}{\epsilon}\right\rfloor,\left\lfloor\frac{y_{1}}{\epsilon}\right\rfloor\right)$ (resp. $\left.\left(\left\lfloor\frac{x_{2}}{\epsilon}\right\rfloor,\left\lfloor\frac{y_{2}}{\epsilon}\right\rfloor\right)\right)$ that corresponds to the square containing point $\left(x_{1}, y_{1}\right)$ (resp. $\left.\left(x_{2}, y_{2}\right)\right)$.

It is now easy to verify that the function $\gamma$ defined above satisfies the first constraint of Problem $B$ :

$$
\begin{aligned}
& \int_{0}^{y_{1}} \int_{x_{1}}^{1} \gamma\left(x_{1}, y_{1}, x_{2}, y_{2}\right) d x_{2} d y_{2} \\
= & \sum_{i>\left\lfloor\frac{x_{1}}{\epsilon}\right\rfloor, j<\left\lfloor\frac{y_{1}}{\epsilon}\right\rfloor} \int_{\epsilon i}^{\epsilon(i+1)} \int_{\epsilon j}^{\epsilon(j+1)} \gamma\left(x_{1}, y_{1}, x_{2}, y_{2}\right) d y_{2} d x_{2} \\
+ & \sum_{i>\left\lfloor\frac{x_{1}}{\epsilon}\right\rfloor, j=\left\lfloor\frac{y_{1}}{\epsilon}\right\rfloor} \int_{\epsilon i}^{\epsilon(i+1)} \int_{\epsilon j}^{y_{1}} \gamma\left(x_{1}, y_{1}, x_{2}, y_{2}\right) d y_{2} d x_{2} \\
+ & \sum_{i=\left\lfloor\frac{x_{1}}{\epsilon}\right\rfloor, j<\left\lfloor\frac{y_{1}}{\epsilon}\right\rfloor} \int_{x_{1}}^{\epsilon(i+1)} \int_{\epsilon j}^{\epsilon(j+1)} \gamma\left(x_{1}, y_{1}, x_{2}, y_{2}\right) d y_{2} d x_{2} \\
+ & \sum_{i=\left\lfloor\frac{x_{1}}{\epsilon}\right\rfloor, j=\left\lfloor\frac{y_{1}}{\epsilon}\right\rfloor}^{\epsilon(i+1)} \int_{x_{1}}^{y_{1}} \gamma\left(x_{1}, y_{1}, x_{2}, y_{2}\right) d y_{2} d x_{2} \\
= & \sum_{i \geq\left\lfloor\frac{x_{1}}{\epsilon}\right\rfloor, j \leq\left\lfloor\frac{y_{1}}{\epsilon}\right\rfloor} y_{\left(\left\lfloor\frac{x_{1}}{\epsilon}\right\rfloor,\left\lfloor\frac{y_{1}}{\epsilon}\right\rfloor\right),(i, j)} \frac{1}{\epsilon^{2}}
\end{aligned}
$$

Since $y$ is a feasible solution for problem $D$ we get that the last expression is at least:

$$
\begin{aligned}
& \frac{1}{\epsilon^{2}} \cdot w_{\left(\left\lfloor\frac{x_{1}}{\epsilon}\right\rfloor,\left\lfloor\frac{y_{1}}{\epsilon}\right\rfloor\right)} \\
\geq & \frac{1}{\epsilon^{2}}\left(\int_{\epsilon\left\lfloor\frac{x_{1}}{\epsilon}\right\rfloor}^{\epsilon\left(\left\lfloor\frac{x_{1}}{\epsilon}\right\rfloor+1\right)} \int_{\epsilon\left\lfloor\frac{y_{1}}{\epsilon}\right\rfloor}^{\epsilon\left(\left\lfloor\frac{y_{1}}{\epsilon}\right\rfloor+1\right)} f(x, y) d y d x+2 \epsilon^{3} \lambda\right)
\end{aligned}
$$

We can now use the Lipschitz-continuity of $f(x, y)$ to lower-bound $f(x, y)$ within any square by $f\left(x_{1}, y_{1}\right)-2 \epsilon \lambda$, 
for any point $\left(x_{1}, y_{1}\right)$ in the square. This way we get:

$$
\begin{aligned}
& \int_{0}^{y_{1}} \int_{x_{1}}^{1} \gamma\left(x_{1}, y_{1}, x_{2}, y_{2}\right) d x_{2} d y_{2} \\
\geq & \frac{1}{\epsilon^{2}}\left(\epsilon^{2}\left(f\left(x_{1}, y_{1}\right)-2 \epsilon \lambda\right)+2 \epsilon^{3} \lambda\right) \\
= & f\left(x_{1}, y_{1}\right)
\end{aligned}
$$

In complete analogy we define $\gamma\left(x_{1}, y_{1}, x_{2}, y_{2}\right)$, for any pair of points $\left(x_{1}, y_{1}\right),\left(x_{2}, y_{2}\right)$, with $\left(x_{2}, y_{2}\right)$ lying in the northwest orthant of $\left(x_{1}, y_{1}\right)$, so that the second constraint of Problem $B$ is also satisfied ${ }^{4}$.

The non-negativity constraint is also obviously satisfied, so we get a feasible solution $\gamma$ for problem $B$. Its cost $S O L(B)$ can be seen to be:

$$
\begin{aligned}
& \int_{0}^{1} \int_{0}^{1} \int_{0}^{y_{1}} \int_{x_{1}}^{1} \gamma\left(x_{1}, y_{1}, x_{2}, y_{2}\right) d x_{2} d y_{2} d x_{1} d y_{1} \\
= & \int_{0}^{1} \int_{0}^{1} \sum_{i^{\prime} \geq\left\lfloor\frac{x_{1}}{\epsilon}\right\rfloor} \sum_{j^{\prime} \leq\left\lfloor\frac{y_{1}}{\epsilon}\right\rfloor} \frac{1}{\epsilon^{2}} \cdot y_{\left(\left\lfloor\frac{x_{1}}{\epsilon}\right\rfloor,\left\lfloor\frac{y_{1}}{\epsilon}\right\rfloor\right),\left(i^{\prime}, j^{\prime}\right)} \\
= & \sum_{i} \sum_{j} \int_{\epsilon i}^{\epsilon(i+1)} \int_{\epsilon j}^{\epsilon(j+1)} \sum_{i^{\prime} \geq i} \sum_{j^{\prime} \leq j} \frac{1}{\epsilon^{2}} \cdot y_{(i, j),\left(i^{\prime}, j^{\prime}\right)} \\
= & \sum_{i} \sum_{j} \sum_{i^{\prime} \geq i} \sum_{j^{\prime} \leq j} y_{(i, j),\left(i^{\prime}, j^{\prime}\right)} \\
= & \sum_{(u, v) \in E} y_{u v} \\
= & O P T(D)
\end{aligned}
$$

so the Lemma follows.

\section{A.5 Proof of Lemma 3.14}

We prove the theorem for $f(x, y)$. The idea is to assume that there is a horizontal segment (with infinite slope) such that for all points lying within distance $\epsilon$ on top of it $f(x, y)=0$, while for all points lying within $\epsilon$ below the segment $f(x, y)>0$. We then show that the length of this segment is at most $O(\epsilon)$, so by having $\epsilon \rightarrow 0$ we get that any boundary of infinite slope has essentially measure zero.

Claim: Fix $y \in[0,1]$ and some $\epsilon, 0 \leq \epsilon \leq 1$. Let $\mathcal{I}=$ $[\bar{x}-\Delta x, \bar{x}] \subseteq[0,1]$ be an interval such that $f(x, y)>0$ and $f(x, y+\epsilon)=0$ for all $x \in \mathcal{I}$. It then holds that $\Delta x=O(\epsilon)$. Proof of Claim: Let $F_{y}(x)=x \cdot \int_{x}^{1} \phi(x, y) d x$. By definition of $f(\cdot, \cdot)$, it is easy to verify that $F_{y}(x)$ is strictly decreasing and $F_{y+\epsilon}(x)$ is (weakly) increasing. We then have:

$$
\begin{aligned}
F_{y}(\bar{x}-\Delta x) & =F_{y}(\bar{x})+\int_{\bar{x}-\Delta x}^{\bar{x}} f(x, y) d x \\
& \geq F_{y}(\bar{x})+\int_{\bar{x}-\Delta x}^{\bar{x}}(f(\bar{x}, y)-\Delta x \cdot \lambda) d x \\
& =F_{y}(\bar{x})+\Delta x \cdot(f(\bar{x}, y)-\Delta x \cdot \lambda)
\end{aligned}
$$

where we used the definition of $f(\cdot, \cdot)$ and the fact that it is Lipschitz continuous for some constant $\lambda$. In this proof we also assume that $f(\bar{x}, y)-\Delta x \cdot \lambda>0$; this assumption is wlog but it greatly simplifies the exposition.

\footnotetext{
${ }^{4}$ Notice that in general $\gamma$ is symmetric, i.e. $\gamma\left(x_{1}, y_{1}, x_{2}, y_{2}\right)=\gamma\left(x_{2}, y_{2}, x_{1}, y_{1}\right)$, except for pairs of points that correspond to pairs of grid-points that are on the same line or column.
}

We also have:

$$
\begin{aligned}
F_{y}(\bar{x}-\Delta x) & \leq F_{y+\epsilon}(\bar{x}-\Delta x)+\epsilon \lambda^{\prime} \\
& \leq F_{y+\epsilon}(\bar{x})+\epsilon \lambda^{\prime} \\
& \leq F_{y}(\bar{x})+2 \epsilon \lambda^{\prime}
\end{aligned}
$$

where in the first and third inequality we used Lipschitz continuity of $F_{y}(\cdot)$ with respect to $y$ and in the second inequality we used the fact that $F_{y}(x)$ is weakly increasing in $x$.

Combining the above two inequalities we get:

$$
\Delta x \leq \frac{2 \epsilon \lambda^{\prime}}{f(\bar{x}, y)-\Delta x \cdot \lambda}=O(\epsilon)
$$

\section{A.6 Proof of Theorem 3.15}

Algorithm 1 returns a valid allocation pair so it is truthful by construction.

Suppose $\alpha(y)$ violates condition (1) for some $y \in[0,1]$; it is easy to show then that $f(\alpha(y), y)=0$. Let $\alpha^{\prime}(y)=$ $\arg \max _{x^{\prime} \geq \alpha(y)} x^{\prime} \int_{x^{\prime}}^{1} \phi(x, y) d x$; we want to argue that $\alpha^{\prime}(y)-$ $\alpha(y) \leq \Theta(\epsilon)$. To do that we consider the boundary node of the MWIS returned by the algorithm whose square on the unit plane contains $y$; since this node belongs to the boundary we can assume wlog that it has non-zero weight, hence there must exist some point $\left(x_{1}, y_{1}\right)$ in the corresponding square on the unit plane with $f\left(x_{1}, y_{1}\right) \neq 0$; on the other hand for $(\alpha(y), y)$ we have $f(\alpha(y), y)=0$. Using this, the fact that $\left|y_{1}-y\right|=\Theta(\epsilon)$ and the bounded slope property for the boundary curve $\alpha^{\prime}(y)$ we conclude that $\left|x_{1}-\alpha^{\prime}(y)\right|=$ $\Theta(\epsilon)$ and so $\left|\alpha(y)-\alpha^{\prime}(y)\right|=\Theta(\epsilon)$. A similar argument applies for $\beta(x)$ and $\beta^{\prime}(x)=\arg \max _{y^{\prime} \geq \beta(x)} y^{\prime} \int_{y^{\prime}}^{1} \phi(x, y) d y$ so the profit of the algorithm is:

$$
\begin{aligned}
& \int_{0}^{1}\left[\alpha(y) \cdot \int_{\alpha(y)}^{1} \phi(x, y) d x\right] d y \\
& +\int_{0}^{1}\left[\beta(x) \cdot \int_{\beta(x)}^{1} \phi(x, y) d y\right] d x \\
\geq & \int_{0}^{1}\left[\left(\alpha^{\prime}(y)-\Theta(\epsilon)\right) \cdot \int_{\alpha^{\prime}(y)}^{1} \phi(x, y) d x\right] d y \\
& +\int_{0}^{1}\left[\left(\beta^{\prime}(x)-\Theta(\epsilon)\right) \cdot \int_{\beta^{\prime}(x)}^{1} \phi(x, y) d y\right] d x \\
= & \int_{0}^{1} \int_{\alpha^{\prime}(y)}^{1} f(x, y) d x d y+\int_{0}^{1} \int_{\beta^{\prime}(x)}^{1} g(x, y) d y d x-\Theta(\epsilon) \\
= & S O L(C)-\Theta(\epsilon) \\
\geq & O P T(A)-\Theta(\epsilon)
\end{aligned}
$$

In terms of running time, we need to compute $f$ and $g$ (approximately over every unit square with error $\Theta(\lambda \epsilon)$ ), discretize and construct the graphs and then solve the MAXIMUM WEIGHT INDEPENDENT SET problem in a bipartite graph with $\Theta\left(1 / \epsilon^{2}\right)$ nodes; all this can be done in time polynomial in $1 / \epsilon$. 